\title{
Effect of Chute Angle on Charging Behavior of Sintered Ore Particles at Bell-less Type Charging System of Blast Furnace by Discrete Element Method
}

\author{
Hiroshi MIO, ${ }^{1,2)}$ Satoshi KOMATSUKI, ${ }^{3)}$ Masatoshi AKASHI, ${ }^{3)}$ Atsuko SHIMOSAKA, \\ Yoshiyuki SHIRAKAWA, ${ }^{3)}$ Jusuke HIDAKA, ${ }^{3)}$ Masatomo KADOWAKI, ${ }^{4)}$ Shinroku MATSUZAKI') and \\ Kazuya KUNITOMO ${ }^{4}$
}

1) Research Center for Advanced Science and Technology, Doshisha University, 1-3 Tatara-miyakodani, Kyotanabe, Kyoto 6100321, Japan. $\quad$ 2) Kyoto Fine Particle Technology, Keihanna Interaction Plaza Inc., 1-7 Hikaridai, Seika-cho, Soraku-gun, Kyoto 619-0237, Japan. $\quad 3$ 3) Department of Chemical Engineering and Materials Science, Doshisha University, 1-3 Tataramiyakodani, Kyotanabe, Kyoto 610-0321, Japan. 4) Environmental and Process Technology Center, Nippon Steel Corporation, 20-1 Shintomi, Futtsu, Chiba 293-8511, Japan.

(Received on November 13, 2008; accepted on February 2, 2009)

\begin{abstract}
The objective of this paper is to analyze the particle behavior at a bell-less top of blast furnace by using Discrete Element Method. The effect of chute angle on the flowing behavior or the particle segregation was discussed. The particles in the chute are centrifuged by the chute rotation with moving toward the outlet. The most of the smallest particles are pressed up against the chute side wall, while the larger ones stay at the outside of clustered particles due to the particle size segregation. The specific charged mass at charging area increases with increasing the number of charges, and the distribution of charged mass spreads toward the center of blast furnace, because the particles slide downward along with the slope of heap. The coke particles are pressed toward the center of blast furnace by the subsequently charged sintered ore particles with increasing the number of charges. The large collapse of coke layer is seen after 16 times charging under $36.9^{\circ}$ and $43.1^{\circ}$ in chute angle. It is found that the chute angle affects not only the particle segregation but also the collapse of coke layer strongly.
\end{abstract}

KEY WORDS: particle size segregation; chute flow; blast furnace; bell-less charging system; Discrete Element Method; ironmaking process; numerical simulation.

\section{Introduction}

A blast furnace is a huge reactor having more than $5000 \mathrm{~m}^{3}$ in volume for producing pig-iron from iron ore particles. Ore and coke particles are being stacked alternately in layer, and the gas having high temperature is blown from tuyeres. Iron ore particles are reduced during descending, and many physical changes and chemical reactions between each phase are undergoing in there. There are three stages of matter, and the blast furnace is one of the most complicated systems. Thus, it is difficult to control it, and people can do it by only few approaches; i.e. the quality of the raw materials, the burden distribution and the air blasting condition. It has been thought that the burden distribution affects the operating efficiency strongly. Because the void fraction, the gas flow distribution and the burden descending behavior depend on the burden distribution, and the particle segregation during charging at the top of blast furnace affects it very much. Hence many researches had been studied about the particle segregation at the charging process. ${ }^{1-7)}$ They gave many informative results, however, it is quite difficult to understand the segregation inside the particle bed, and grasp the segregating behavior in real- time. The particle segregation occurs at the top of blast furnace as well as at the surge hopper, the conveyor, the bunker and the chute. Therefore, the computational simulation using Discrete Element Method $(\mathrm{DEM})^{8)}$ is required for the estimation and optimization of the charging processes. ${ }^{9-11)}$ DEM is one of the most reliable simulation methods for analyzing the solid particle behavior, however there is one big problem to solve before applying it to the ironmaking process. The particles in DEM are usually assumed to be spherical, although the shapes of ore and coke particles are not spherical. Thus, it is necessary to be able to consider the effect of particle shape on the granular flow in DEM. The authors have proposed the method to solve this issue; that each particle has its own rolling friction. ${ }^{12)}$ The particle behavior and the segregation during flowing through the laboratory-scale chute analyzed by the simulation agreed with the experimental results very well, and its agreement could be seen under several different conditions (chute angle, installation of a damper or particle mixture). ${ }^{12)}$ Therefore, this simulation method has a high potential to analyze the particle segregation during charging to the actual blast furnace.

In this paper, the particle behavior of sintered ore at the 
bell-less top of blast furnace was analyzed by using DEM that was able to consider the effect of particle shape on granular flow. The effect of chute angle on the charging behavior and the particle segregation at the charged bed were discussed.

\section{Simulation}

Discrete Element Method (DEM) ${ }^{8)}$ is one of the most popular and reliable simulation methods for the numerical analysis of solid particle behavior. This simulation method consists of the idea of determining the kinematic force to each finite-sized particle. The main calculation of DEM consists three steps; i.e. 1) particle detection, 2) calculation of forces, 3) update of trajectories, and these processes are looped until $t=t_{\max }$. The particle detection process has the heaviest calculation load in DEM. ${ }^{13)}$ The speed-up of particle detection process can be possible by optimization of cell condition. ${ }^{13-15)}$ The contact between two particles is given by Voigt model, which consists of a spring-dashpot and a slider for the friction in the tangential component. The contact forces, $\mathbf{F}_{n}$ and $\mathbf{F}_{t}$, are calculated by following equations.

$$
\begin{array}{r}
\mathbf{F}_{n, i j}=\left(K_{n} \Delta u_{n, i j}+\eta_{n} \frac{\Delta u_{n, i j}}{\Delta t}\right) \mathbf{n}_{i j} \ldots \ldots \ldots \ldots \ldots . . . . .(1) \\
\mathbf{F}_{t, i j}=\min \left\{\mu\left|\mathbf{F}_{n, i j}\right|_{i j},\left[K_{t}\left(\Delta u_{t, i j}+\Delta \phi_{i j}\right)+\eta_{t}\left(\frac{\Delta u_{t, i j}+\Delta \phi_{i j}}{\Delta t}\right)\right] \mathbf{t}_{i j}\right\}
\end{array}
$$

Where, $K$ and $\eta$ mean the spring and the damping coefficients. $\Delta u$ and $\Delta \phi$ are a relative translational displacement of gravitational center between two particles and a relative displacement at the contact point caused by the particle rotation. $\mu$ is the frictional coefficient (particle-particle: 0.43 , particle-wall: 0.58 ). The frictional coefficients of particle were estimated by shearing test of the single particles and comparing particle behavior, and the particle behaviors using these values had been validated with the actual ones in the previous report. ${ }^{12)}$ Thus, these values were used in this work. $\mathbf{n}_{i j}$ and $\mathbf{t}_{i j}$ denote the unit vector from the $i$-th particle to the $j$-th one in the normal and the tangential components. The subscript $n$ and $t$ also denote the normal and the tangential components. The translational and rotational motions of each particle are updated by following equations.

$$
\begin{gathered}
\boldsymbol{k}=\frac{\sum \mathbf{F}}{m}+\mathbf{g} \\
\boldsymbol{\omega}=\frac{\sum \mathbf{M}}{I}
\end{gathered}
$$

Where, $\mathbf{v}$ is the vector of a particle velocity, $\mathbf{F}$ is the contact force acting on a particle, $m$ and $\mathbf{g}$ mean the mass of a particle and gravitational acceleration, $\boldsymbol{\omega}$ is the vector of angular velocity, $\mathbf{M}$ and $I$ denote the moment caused by the tangential force and the moment of inertia. The shape of particle in DEM is usually assumed to be spherical due to the ease of contact detection or calculation of contact force, although the shape of sintered ore particle is completely ir- regular. Thus, its effect on the particle motion must be considered in DEM by setting the proper rolling friction for each particle, and it is given in following equation. ${ }^{11)}$

$$
\mathbf{M}_{r, i}=-\frac{3}{8} \alpha_{i} b\left|\mathbf{F}_{n}\right| \frac{\boldsymbol{\omega}_{i}}{\left|\boldsymbol{\omega}_{i}\right|}
$$

Where, $b$ is a radius of contact area, and $\alpha_{i}$ denotes the coefficient of rolling friction. Every particle has different $\alpha_{i}$, because the shapes of sintered ore particles are totally different from each other. Its distribution is related with the rollability of particle. ${ }^{12)}$ The method having individual rolling friction had given the significant agreement with the experimental results, ${ }^{12}$ therefore, this method was applied to the present work.

Figure 1 shows the bell-less type charging system of the blast furnace, which was modeled in this work. The length of horseshoe-shaped chute were about $5.3 \mathrm{~m}$ and, and a damper ${ }^{16)}$ was installed at the outlet of the chute with $26^{\circ}$ (Fig. 2). 31 tons (620231 particles, density, $\rho: 3300 \mathrm{~kg} / \mathrm{m}^{3}$, Young's modulus, E: $35 \mathrm{GPa}$, Poisson's ratio, $v: 0.25)$ of sintered ore were positioned in the hopper randomly and packed. The particle ratio of smallest particle at the bottom was set bit larger than others to relate to the actual result. The coke layer was made at the top of blast furnace in advance, as shown in Fig. 1, and its surface profile was in

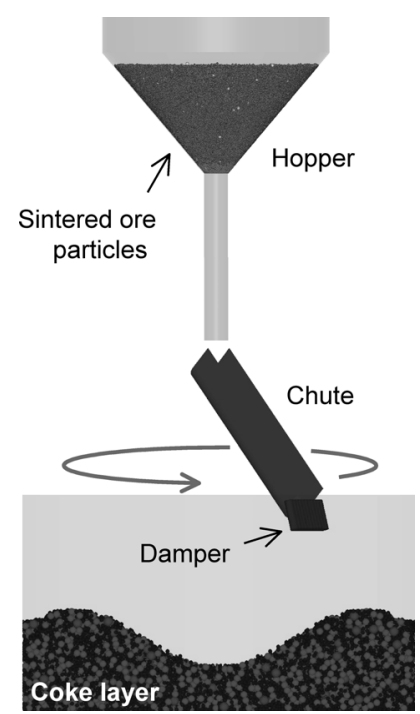

Fig. 1. Schematic diagram of bell-less charging system that modeled in this work.

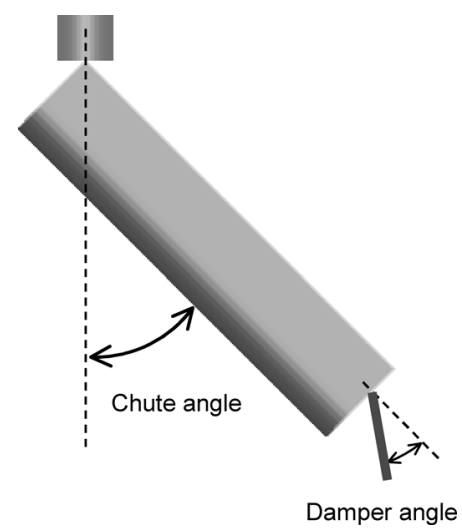

Fig. 2. Schematic diagram of chute and damper conditions. 
Table 1. Number of particles for sintered ore.

\begin{tabular}{ccc}
\hline $\begin{array}{c}\text { Diameter } \\
{[\mathrm{mm}]}\end{array}$ & $\begin{array}{c}\text { Mass ratio } \\
{[\mathrm{wt} \%]}\end{array}$ & $\begin{array}{c}\text { Number of particles } \\
{[-]}\end{array}$ \\
\hline 22.5 & 30.2 & 474946 \\
37.5 & 34.1 & 115848 \\
52.5 & 17.8 & 22126 \\
67.5 & 9.8 & 5743 \\
97.5 & 8.1 & 1568 \\
\hline
\end{tabular}

Table 2. Number of particles for coke.

\begin{tabular}{ccc}
\hline $\begin{array}{c}\text { Diameter } \\
{[\mathrm{mm}]}\end{array}$ & $\begin{array}{c}\text { Mass ratio } \\
{[\mathrm{wt} \%]}\end{array}$ & $\begin{array}{c}\text { Number of particles } \\
{[-]}\end{array}$ \\
\hline 67.5 & 1.7 & 14292 \\
112.5 & 44.8 & 79973 \\
187.5 & 44.7 & 17216 \\
262.5 & 8.8 & 1231 \\
\hline
\end{tabular}

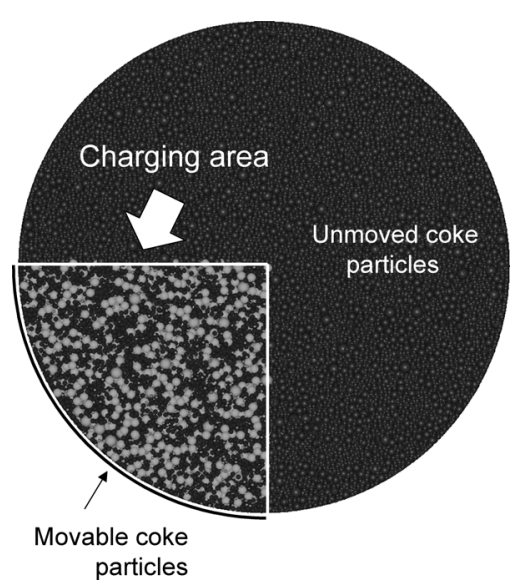

Fig. 3. Schematic diagram of charging area.

agreement with the actual one. The number of coke particles was $112712\left(\rho: 1050 \mathrm{~kg} / \mathrm{m}^{3}, E: 5.4 \mathrm{GPa}, v: 0.22\right)$. The particle size distributions of sintered ore and coke were related to actual ones, however the particle sizes were adjusted relatively three times bigger than actual ones to reduce the number of calculated particles. The detailed particle conditions are tabulated in Tables $\mathbf{1}$ and $\mathbf{2}$. The charging area of sintered ore particles from the chute was limited to the area of quarter of blast furnace due to the reduction of calculation time (Fig. 3). The chute was returned every $90^{\circ}$ of rotation, and it stopped temporary to wait the particle sliding on the charged layer when it returned. The coke particles at the charging area and in the layer thickness of about $1 \mathrm{~m}$ from the surface can be moved, which is shown in Fig. 4, while the other coke particles cannot move. That is to say;

- Contact detections between the unmoved coke particles

- Calculations of contact force between the unmoved coke particles

- Calculations of trajectories for the unmoved coke particles

were ignored for speeding-up the calculation. The contact force between the movable particles (sintered ore or coke) and the unmoved coke particles were, of course, calculated, thus the information of unmoved particle should be stored in the searching cell. The chute angle, $\theta_{\mathrm{c}}$ : Fig. 2, was changed as $36.9^{\circ}, 43.1^{\circ}$ or $49.0^{\circ}$, and the rotational speed

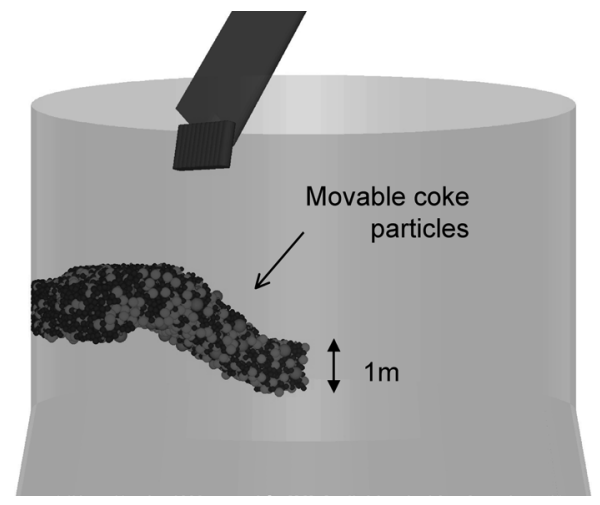

Fig. 4. Schematic diagram of movable coke particles.

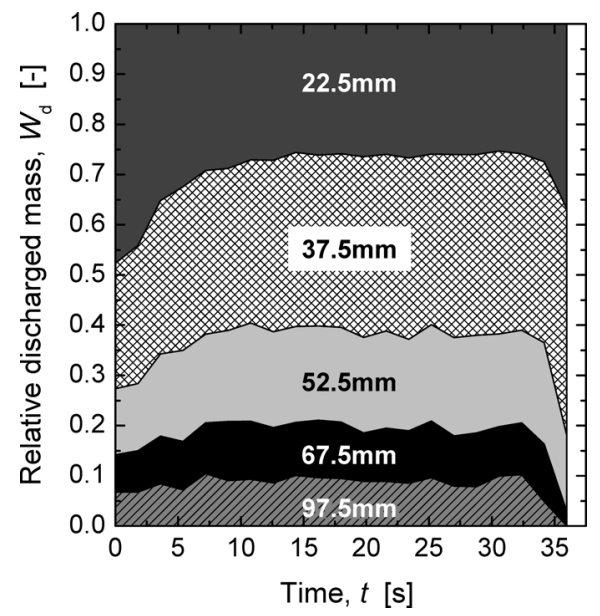

Fig. 5. Relation between the relative discharged mass of sintered ore from the hopper and the discharging time.

was $8.0 \mathrm{rpm}$. The sintered ore particles were charged in the blast furnace 16 times, and the chute angle wasn't changed during rotating in this analysis. The coke layer in the blast furnace didn't descend during charging, and the effect of gas flow was not considered. $\Delta t$ was $1.75 \mu \mathrm{s}$, and the total number of calculation steps was about 28 millions steps. All calculations were executed by parallel computing using OpenMP. ${ }^{17)}$

\section{Results and Discussions}

\subsection{Analysis of Particle Behavior during Flowing in the Chute}

Figure 5 shows the relation between the relative discharged mass of sintered ore from the hopper and the discharging time. It is found that the ratio of smallest particle at the early stage is large, because the smallest particle was located at the bottom of hopper more than other sizes of particles in advance to relate the actual phenomenon. On the other hand, the relative masses are mostly constant after 10 s. Figure 6 shows the typical snapshot of particle flowing behavior during charging at the bell-less top of blast furnace under $\theta_{\mathrm{c}}=43.1^{\circ}$, and the sintered ore particles are being charged on the coke layer. Figure 7 shows the snapshots of cross section in the chute; $L / L_{0}=0.5,0.75$ or 1.0 (see Fig. 6). The different colors of particles designate the particle size (brown: $22.5 \mathrm{~mm}$, pink: $37.5 \mathrm{~mm}$, orange: $52.5 \mathrm{~mm}$, red: $67.5 \mathrm{~mm}$ and yellow: $97.5 \mathrm{~mm}$ ). It is found from these snapshots that the particles are centrifuged by 


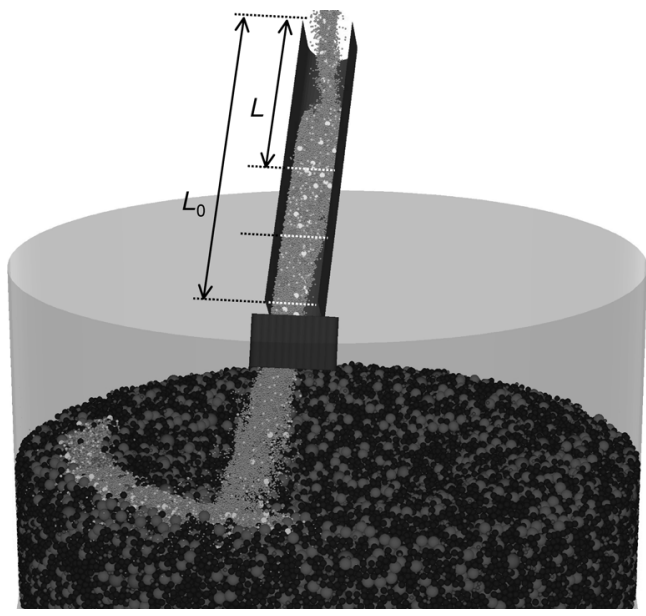

Fig. 6. Typical snapshot of particle flowing behavior during charging under $\theta_{\mathrm{c}}=43.1^{\circ}$.

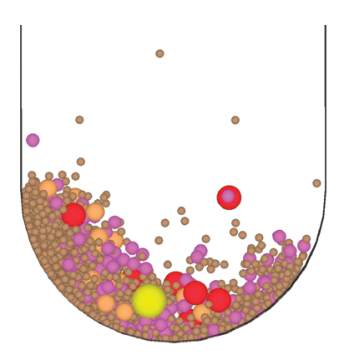

(a) $L / L_{0}=0.5$

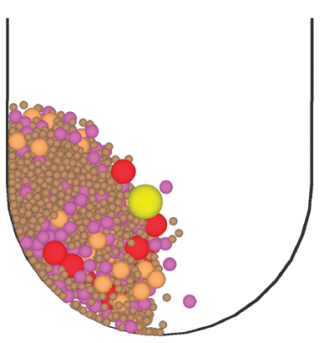

(c) $L / L_{0}=1.0$

Fig. 7. Snapshots of cross section in the chute under $\theta_{\mathrm{c}}=43.1^{\circ}$.

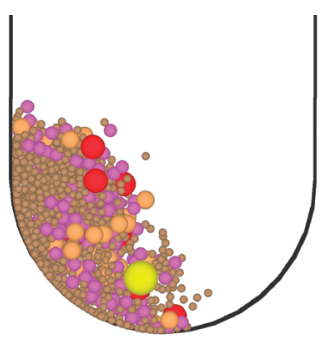

(a) Chute angle : $36.9^{\circ}$

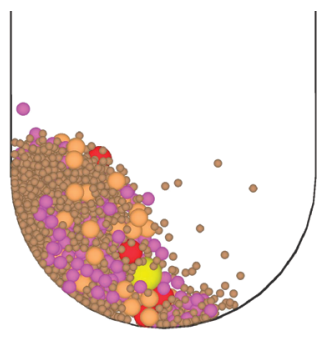

(b) $L / L_{0}=0.75$
Fig. 8. Snapshots of cross section in the chute at $L / L_{0}=1.0$.

the chute rotation with moving toward the outlet. The most of the smallest particles are pressed up against the chute side wall at $L / L_{0}=1.0$, while the larger ones stay at the outside of clustered particles due to the particle size segregation. The segregation has already occurred before being charged into the blast furnace. Figure 8 shows the snapshots of the cross section at $L / L_{0}=1.0$ for other two differ-

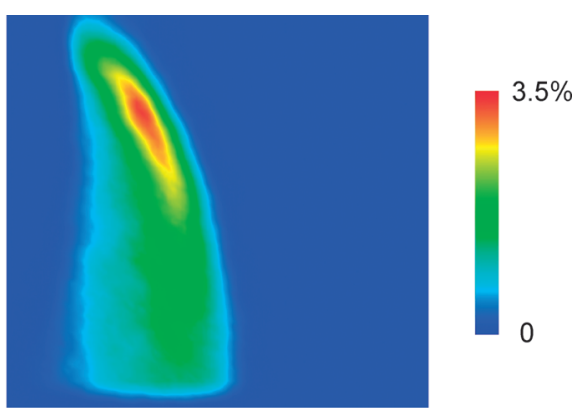

(a) $22.5 \mathrm{~mm}$

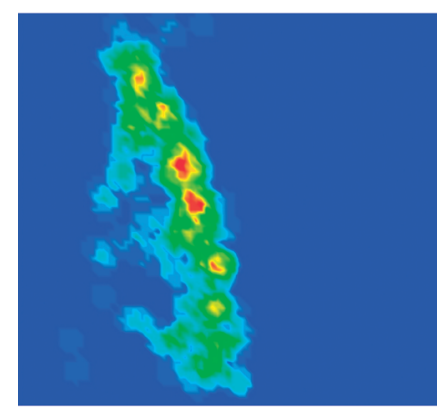

(b) $97.5 \mathrm{~mm}$

Fig. 9. Mapping of impact frequency of discharging particles on the damper under $\theta_{\mathrm{c}}=43.1^{\circ}$.

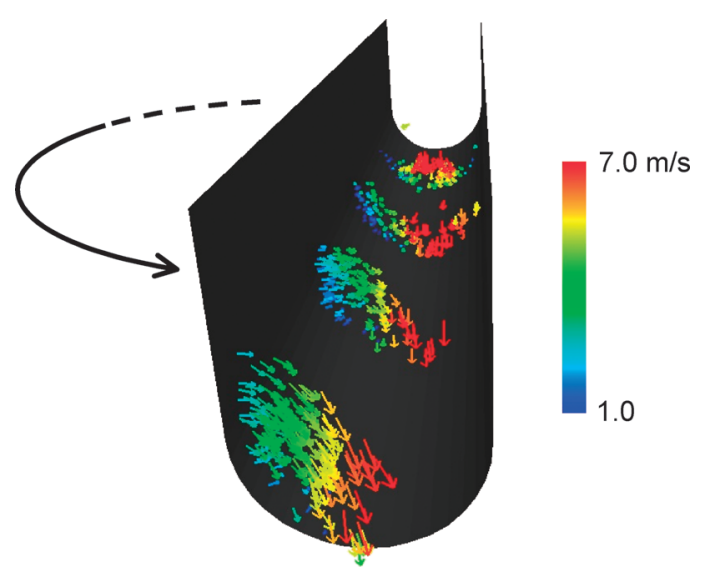

Fig. 10. Vector of particle velocity in the chute.

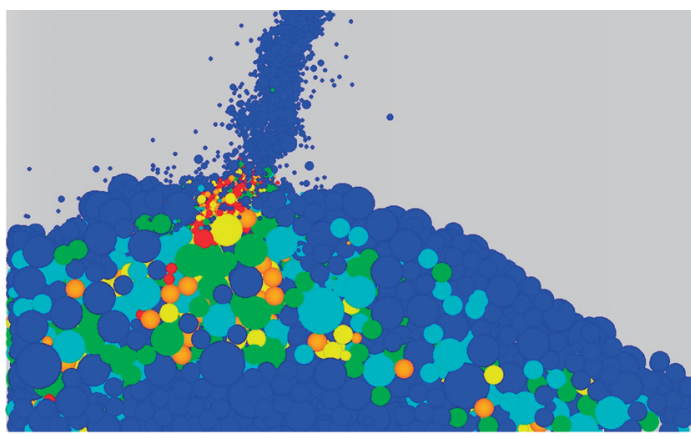

$$
F_{i} / F_{g, i}=10^{1.25}
$$

Fig. 18. Snapshot of cross section of particle layer colored by the degree of contact force at first charging. 
ent conditions $\left(\theta_{\mathrm{c}}=36.9^{\circ}\right.$ or $\left.49.0^{\circ}\right)$. The amount of particles in the chute decreases with decreasing the chute angle. The particles have larger velocity because the gravitational acceleration becomes more dominant when the chute angle is small, then they are discharged easily. The tendency of particle size segregation in the chute is quite similar under the different chute angles, because the shape of chute and its rotational speed are more dominant for the segregation in the chute. Figure 9 shows the mapping of impact frequency of discharging particles on the damper under $\theta_{\mathrm{c}}=43.1^{\circ}$. The outer ward of this mapping denotes the dimension of the damper. The smallest particles hit on the upper area of the damper, on the other hand, the largest one hits on the middle. These tendencies are closely related to the particle size segregation in the chute, as shown in Fig. 7. Figure 10 shows the vector of particle velocity in the chute for four areas $\left(L / L_{0}=0.25,0.5\right.$. 0.75 or 1.0$)$ from the view point of the outlet. The particles near the right wall have much larger velocities than those near left wall $\left(L / L_{0}=0.5\right)$, because they are accelerated by centrifugal force and the arc of the chute right wall. It is found that the particle motion at the outlet is like tumbling (Fig. 11). The particles at the right side $\left(L / L_{0}=0.25\right)$ move toward the left wall with high velocity, and they slide into the space between the particle and wall. Then, the particles, especially smaller ones, are pressed up against the wall, and some of them fall down by the gravitational force. Thus, it is thought that the particle flow in the chute is kind of twisted.

\subsection{Analysis of Particle Segregation of Charged bed}

Figure 12 shows the snapshots of the cross section of charged bed at 4 th, 8 th, 12th or 16th charging processes under $43.1^{\circ}$. The sintered ore particles are being charged on the coke layer with chute rotation, and the particles are stacked at the charging area. Figure 13 shows the relation between the specific charged mass (the charged mass per area), $W_{\mathrm{s}}$, and the radial distance from the center of the blast furnace, $L_{\mathrm{R}}$, with a parameter of the number of charges. $W_{\mathrm{s}}$ at charging area increases with increasing the number of charges, and the distribution of $W_{\mathrm{s}}$ spreads toward the center. Figure 14 shows the relation between $W_{\mathrm{s}}$ for every 4 charges and $L_{\mathrm{R}}$. It is found that the particles are charged toward the center of blast furnace with increasing the number of charges significantly, because the particles slide downward along with the slope of the heap. Figure 15 shows the relations between the specific charged mass for each sized particle and $L_{\mathrm{R}}$ after 16 times charging. The mass distribution is given wider as the particle size becomes larger. Figure 16 also shows the relation between the relative specific charged mass for each sized particle, $W_{\mathrm{r}}$, and $L_{\mathrm{R}}$, and the dashed lines in this figure denote the initial mass ratio of particles. It is found that the most of the smallest particles are positioned at the center of the charged bed because the small particles percolate in the particle bed easily, then most of them don't slide on the heap. On the other hand, the larger particles are deposited at both sides of charged bed because the large particles can slide and bounce over the hole easily on the particle bed. Even so, about $94 \mathrm{wt} \%$ of charged particles are at $L_{\mathrm{R}}=2.6-5.2 \mathrm{~m}$, and the particle mass ratio at this range is close to the initial level, thus, the particle size segregation is not so strong in

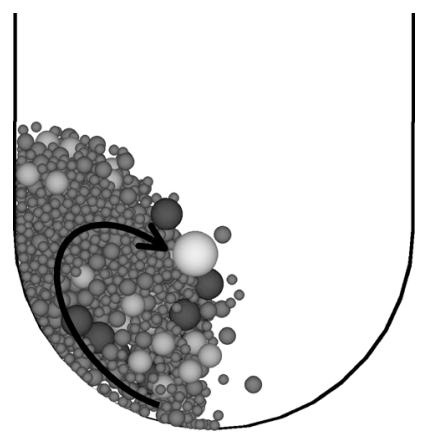

Fig. 11. Schematic diagram of particle motion at the outlet.

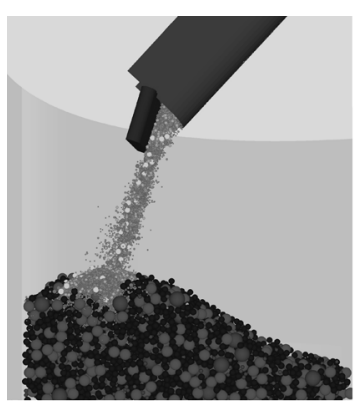

(a) 4th charging

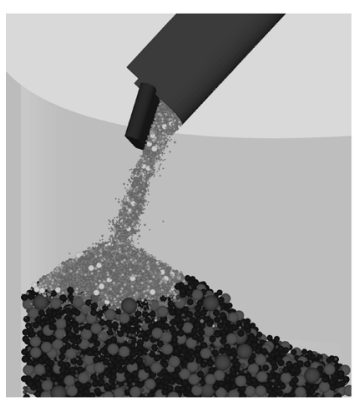

(c) 12th charging

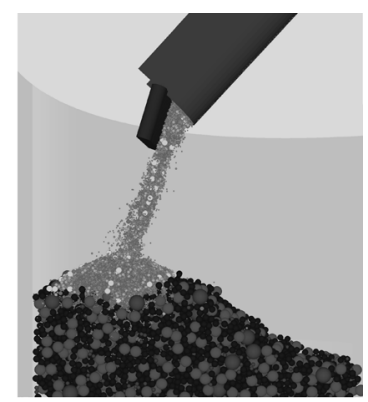

(b) 8th charging

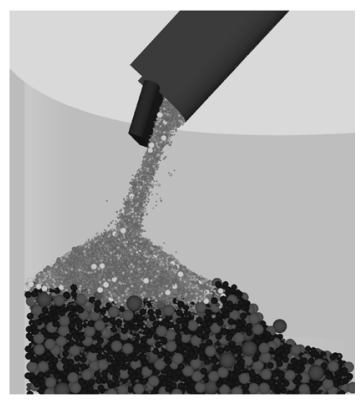

(d) 16th charging
Fig. 12. Snapshots of the cross section of charged bed under $\theta_{\mathrm{c}}=43.1^{\circ}$

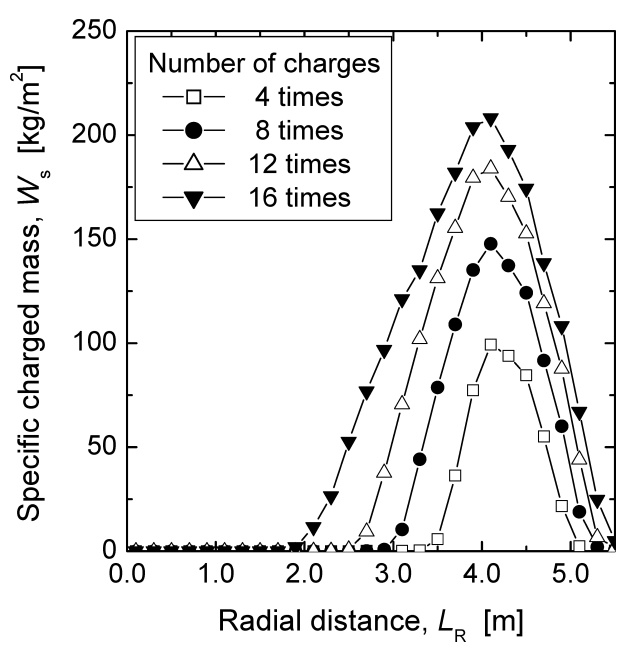

Fig. 13. Relation between the specific charged mass and the radial distance under $\theta_{\mathrm{c}}=43.1^{\circ}$.

this condition. Figure 17 shows the snapshots of coke layer after charging processes. The line in each snapshot denotes the initial profile of coke layer. The coke particles are 


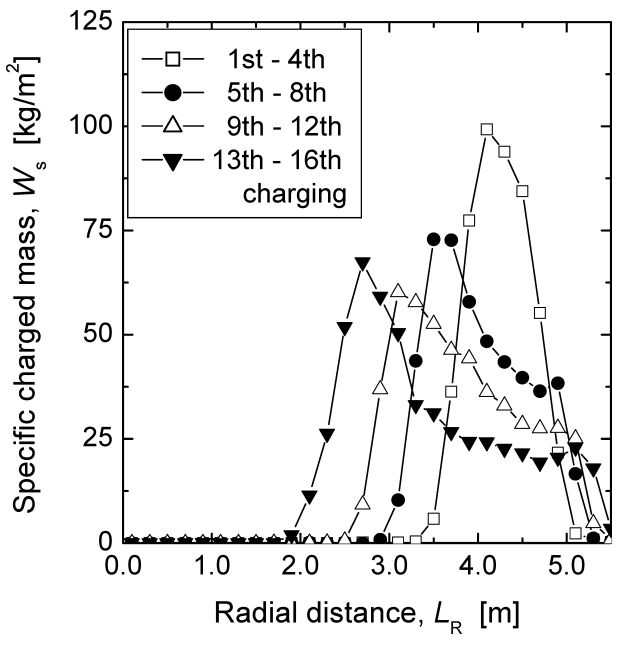

Fig. 14. Relation between the specific charged mass for every 4 charges and the radial distance under $\theta_{c}=43.1^{\circ}$.

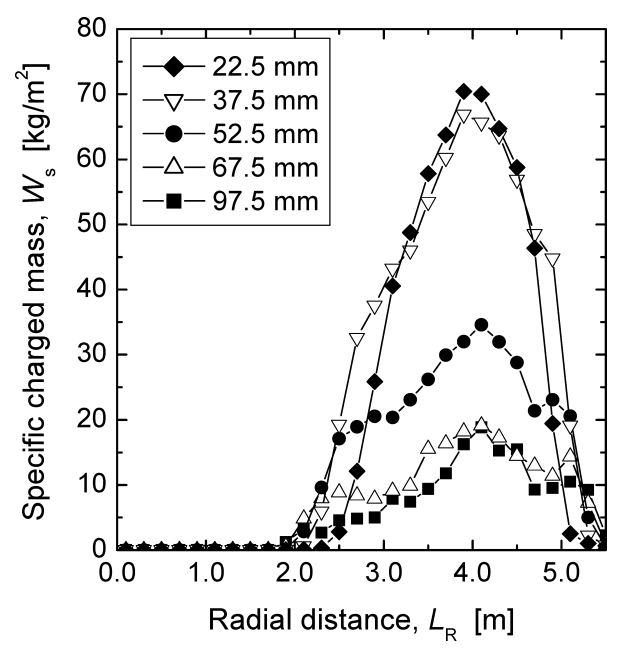

Fig. 15. Relation between the specific charged mass for each sized particle and the radial distance after 16 times charging under $\theta_{\mathrm{c}}=43.1^{\circ}$.

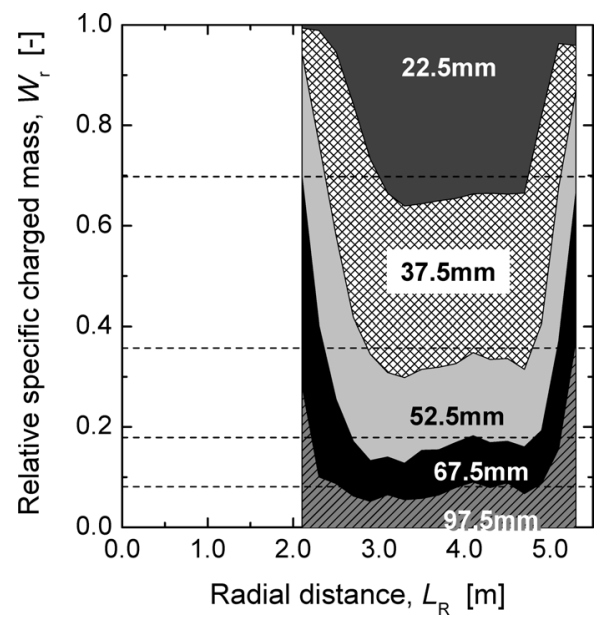

Fig. 16. Relation between the relative charged mass for each sized particle and the radial distance after 16 times charging under $\theta_{\mathrm{c}}=43.1^{\circ}$.

pressed toward the center of blast furnace by the charged sintered ore particles with increasing the number of charges. The large collapse of coke layer is seen after 16 times charging, and $0.4 \mathrm{~m}$ in depth of collapse is found. Be-

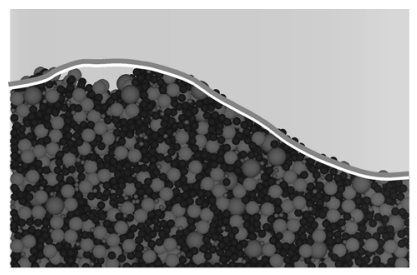

(a) After 4 times charging

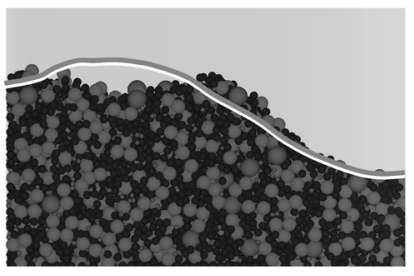

(c) After 12 times charging

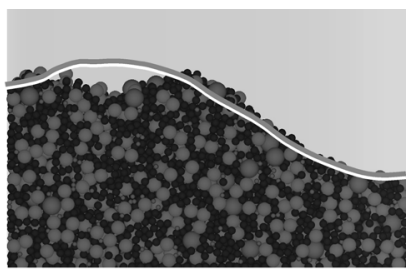

(b) After 8 times charging

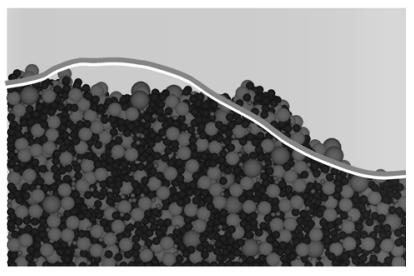

(d) After 16 times charging
Fig. 17. Snapshots of coke layer after charging process under $\theta_{\mathrm{c}}=43.1^{\circ}$

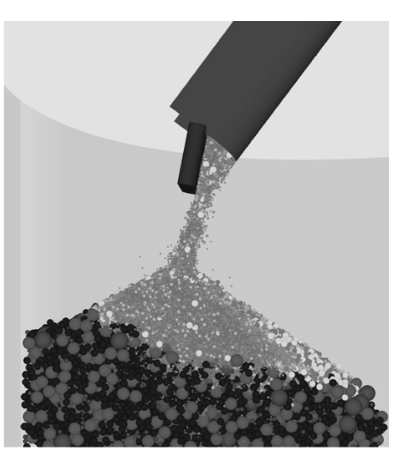

(a) Chute angle : $36.9^{\circ}$

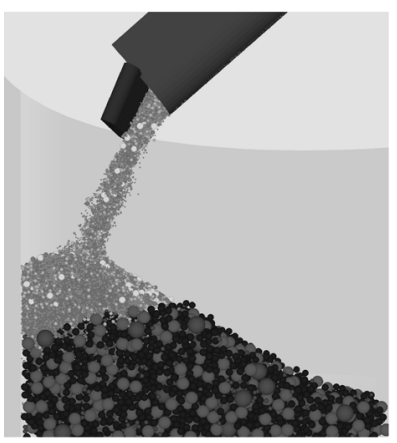

(b) Chute angle : $49.0^{\circ}$
Fig. 19. Snapshots of cross section of charged bed at 16th charging.

cause the sintered ore particle has about 3 times larger density than that of coke particle, and the particle size of ore is much smaller than coke, thus the ore particle thrusts in the coke layer easily. Figure 18 shows the snapshots of cross section of particle layer at 1 st charging, and the particles are colored according to the relative contact force acting on the particle. The contact force is normalized by the gravitational force of each particle, because the difference in the particle size is too large. The range of colors in this figure is logarithmic order. The contact forces at the charging area are significantly larger than others. This large contact force acts on the coke layer as "wedge", and the coke particle are pressed toward both sides.

Figure 19 shows the snapshots of the cross section of 


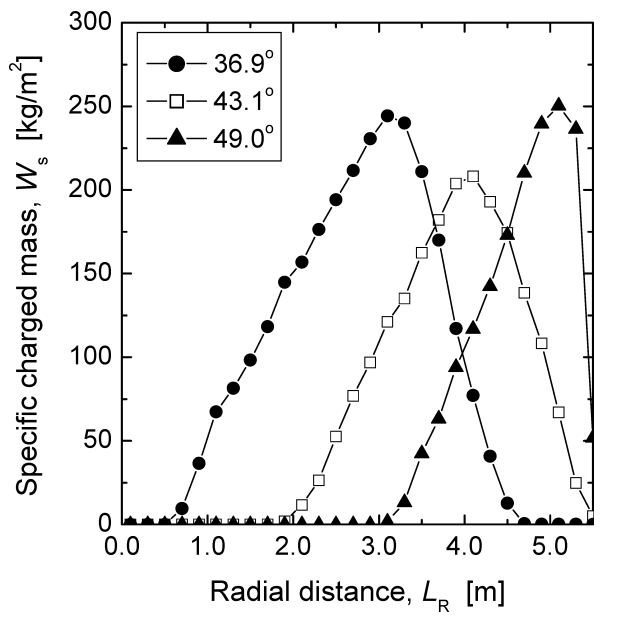

Fig. 20. Relation between the specific charged mass and the radial distance with a parameter of chute angle after 16 times charging.

charged bed at 16 th charging under $\theta_{c}=36.9^{\circ}$ or $49.0^{\circ}$. A lot of sintered ore particles, especially the larger ones, are located near the center of blast furnace under $\theta_{\mathrm{c}}=36.9^{\circ}$, on the other hand, most of them are charged at the terrace of coke layer under $\theta_{c}=49.0^{\circ}$. The position of the heap of charged particles is according to the chute angle, and this is also confirmed from Fig. 20, which shows the relation between the specific charged mass, $W_{\mathrm{s}}$, and the radial distance, $L_{\mathrm{R}}$, with a parameter of chute angle after 16 times charging. The peak of the mass of charged particles shifts toward the center of blast furnace with decreasing the chute angle. Figure 21 shows the relation between $W_{\mathrm{s}}$ for every 4 charges and $L_{\mathrm{R}}$ under $\theta_{\mathrm{c}}=36.9^{\circ}$ or $49.0^{\circ}$. It is found that the particles start to slide toward the center of blast furnace with increasing the number of charges, even the particles are charged at the terrace $\left(\theta_{c}=49.0^{\circ}\right)$. The particles can slide easily when the chute angle is small, because the particles are charged at the side slope of the heap. The distribution after 9th charging is very wider comparing to other conditions. Figure 22 shows the relation between the relative specific charged mass for each sized particle, $W_{\mathrm{r}}$, and $L_{\mathrm{R}}$ for $\theta_{\mathrm{c}}=36.9^{\circ}$ or $49.0^{\circ}$. A lot of smaller particles are at the center of charged bed under $\theta_{\mathrm{c}}=36.9^{\circ}$, and it is found that nearly $80 \mathrm{wt} \%$ of charged particles at $L_{\mathrm{R}}=2.0-2.5 \mathrm{~m}$ is the particles, whose sizes are smaller than $37.5 \mathrm{~mm}$. On the other hand, there are quite few smallest particles at $L_{\mathrm{R}}<1.5 \mathrm{~m}$, and the most of particles are large. The particles can slide easily under this condition, because the particles are charged at the side slope of the heap, as described earlier. The segregation usually occurs when the granular materials flow, and the strong particle size segregation is seen in this condition. The segregation under $\theta_{\mathrm{c}}=49.0^{\circ}$ seems to be less than that of $\theta_{\mathrm{c}}=36.9^{\circ}$, because most of particles are charged at the terrace, as shown in Fig. 19. Although the mass ratio of larger particles at $L_{\mathrm{R}}<3.5 \mathrm{~m}$ is large due to the sliding particle, this segregation affects little. The total mass of charged particles around this area is very small. Figure 23 shows the snapshots of coke layer after 16 times charging. The large collapse of coke layer is found under $\theta_{\mathrm{c}}=36.9^{\circ}$, and thickness of coke layer near center is increased by the collapse. On the other hand, the coke layer keeps initial profile under $\theta_{\mathrm{c}}=49.0^{\circ}$. Thus, the strong parti-

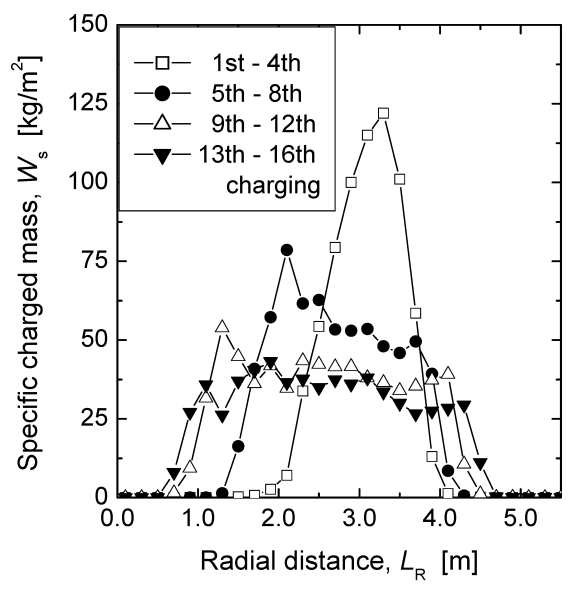

(a) Chute angle : $36.9^{\circ}$

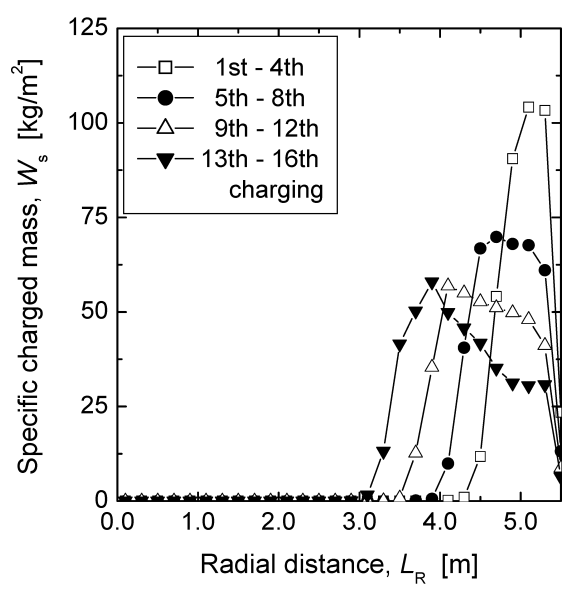

(b) Chute angle : $49.0^{\circ}$

Fig. 21. Relation between the specific charged mass for every 4 charges and the radial distance.

cle segregation and the large collapse occur when the chute angle is small. It is concluded that the burden distribution in the blast furnace is affected by the chute angle.

There is no doubt that DEM is useful for the analysis or estimation of the particle segregation at the charging processes, however there are still important issues to improve it. Although, the calculation speed of DEM cord that authors had developed is faster than that of normal cord, it is still lack of computing power. Hence the particle size in this work was adjusted, and the charging area was limited. This assumption might affect the particle segregation and the collapse of coke layer. The modeling of ironmaking process by DEM is required extremely high computing power, because there are billions and billions particles and the time scale of the operation is too long. Therefore, it's absolutely essential to develop the super large scale DEM algorithm by using parallel computing in the near future.

\section{Conclusions}

In this paper, the effect of chute angle on the charging behavior of sintered ore particle at the bell-less charging system of blast furnace was investigated by using Discrete Element Method. The followings are summaries of this 


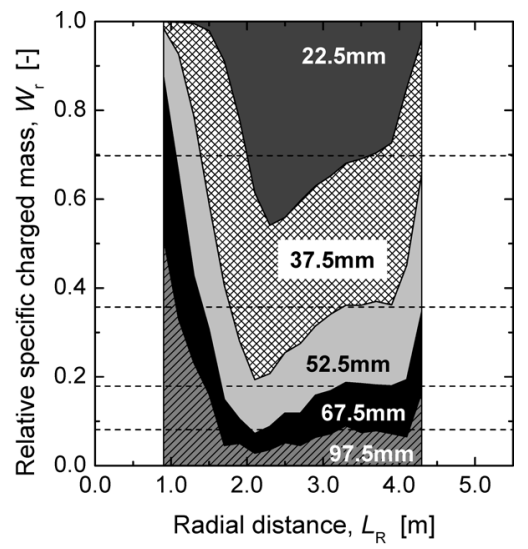

(a) Chute angle : $36.9^{\circ}$

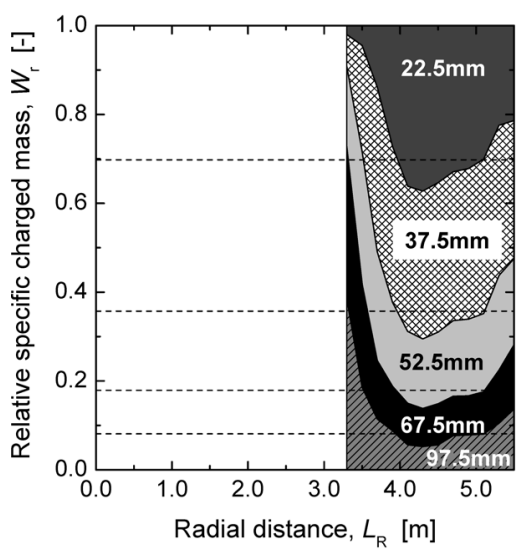

(b) Chute angle : $49.0^{\circ}$

Fig. 22. Relation between the relative specific charged mass for each sized particle and the radial distance after 16 times charging.

work.

(1) The particles in the chute are centrifuged by the chute rotation with moving toward the outlet. The most of the smallest particles are pressed up against the chute side wall, while the larger ones stay at the outside of clustered particles due to the particle size segregation. It is found that the segregation has already occurred before being charged in the blast furnace.

(2) The specific charged mass at charging area increases with increasing the number of charges, and the distribution of charged mass spreads toward the center of blast furnace, because the particles slide downward along with the slope of heap. The sliding behavior of charged particles becomes significant with decreasing the chute angle, and it leads to strong particle size segregation.

(3) The coke particles are pressed toward the center of blast furnace by the charged sintered ore particles with increasing the number of charges. The large collapse of coke layer is seen after 16 times charging under $\theta_{\mathrm{c}}=36.9^{\circ}$ and $43.1^{\circ}$. Because the sintered ore particle has about 3 times larger density than that of coke particle, and the particle

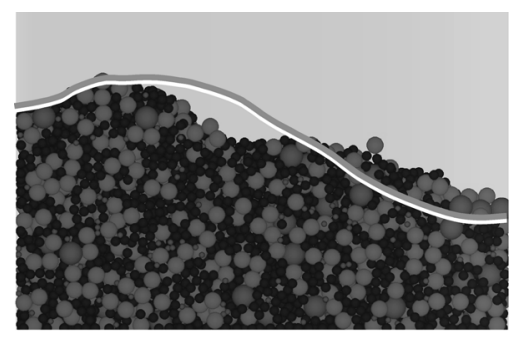

(a) Chute angle : $36.9^{\circ}$

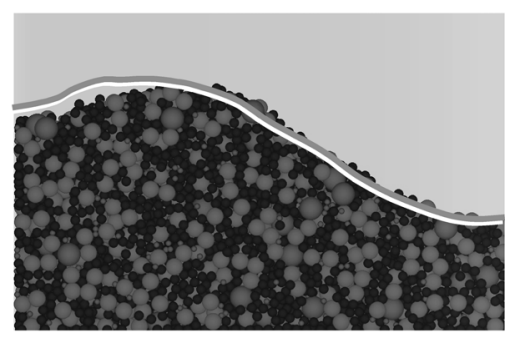

(b) Chute angle : $49.0^{\circ}$

Fig. 23. Snapshots of cross section of coke layer after 16 times charging.

size of ore is smaller than coke, thus the ore particle thrusts in the coke layer easily.

\section{REFERENCES}

1) Y. Kajiwara, T. Jimbo, T. Joko, Y. Aminaga and T. Inada: Tetsu-toHagané, 71 (1985), 175.

2) Y. Okuno, K. Kunitomo, T. Irita and S. Matsuzaki: Tetsu-to-Hagané, 72 (1986), 783.

3) Y. Okuno, S. Matsuzaki, K. Kunitomo, M. Isoyama and Y. Kusano: Tetsu-to-Hagané, 73 (1987), 91.

4) T. Inada, Y. Kajiwara and T. Tanaka: ISIJ Int., 29 (1989), 761.

5) T. Sawada, T. Uetani, S. Taniyoshi, M. Miyagawa, H. Sugawara and M. Yamazaki: Tetsu-to-Hagané, 71 (1992), 1337.

6) M. Hattori, B. Iino, A. Shimomura, H. Tsukiji and T. Ariyama: Tetsu-to-Hagané, 78 (1992), 1345.

7) T. Sato, T. Nouchi, K. Takeda and H. Kamano: Tetsu-to-Hagané, 86 (2000), 648.

8) P. A. Cundall and O. D. L. Strack: Geotechnique, 29 (1979), 47.

9) T. Tanaka, Y. Kajiwara and T. Inada: Tetsu-to-Hagané, 74 (1988), 2262.

10) Y. Kajiwara, T. Inada and T. Tanaka: Tetsu-to-Hagané, 75 (1989), 235.

11) S. Matsuzaki and Y. Taguchi: Tetsu-to-Hagané, 88 (2002), 823.

12) H. Mio, S. Komatsuki, M. Akashi, A. Shimosaka, Y. Shirakawa, J. Hidaka, M. Kadowaki, S. Matsuzaki and K. Kunitomo: ISIJ Int., 48 (2008), 1696.

13) H. Mio, A. Shimosaka, Y. Shirakawa and J. Hidaka: J. Chem. Eng. Jpn., 38 (2005), 969.

14) H. Mio, A. Shimosaka, Y. Shirakawa and J. Hidaka: J. Chem. Eng. Jpn., 39 (2006), 409.

15) H. Mio, A. Shimosaka, Y. Shirakawa and J. Hidaka: Adv. Powder Technol., 18 (2007), 441.

16) A. Matsui, K. Kakiuchi, K. Akagi, S. Matsuzaki and H. Ootsuka: 1998 ICSTI/Ironmaking Conference Proc., AIME, ISS, Warrendale, PA, (1998), 333.

17) R. Chandra, L. Dagum, D. Kohr, D. Maydan, J. McDonald and R. Menon: Parallel Programming in OpenMP, Morgan Kaufmann Publishers, San Francisco, (2001). 